\title{
Audio Quality Measurements for Wireless Microphones in Spectrum Pooling Scenarios
}

\author{
Martin Fuhrwerk, Christoph Thein, and Jürgen Peissig \\ Institute of Communications Technology (IKT) \\ Leibniz Universität Hannover \\ Appelstr. 9a, 30167 Hannover, Germany \\ \{fuhrwerk, thein, peissig\}@ikt.uni-hannover.de
}

\begin{abstract}
In this contribution the influence of different broadband OFDM schemes on the perceptual audio quality of narrowband wireless microphone links is evaluated, since coexistence scenarios of wireless microphones and Orthogonal frequencydivision multiplexing (OFDM) based services arise in the TV bands. Therefore, we present different non-contiguous cyclicprefix (CP-)OFDM and offset quadrature amplitude modulation (OQAM-)OFDM system designs based on the spectrum pooling concept. We measure their power suppression in the subchannel allocated for the wireless microphone. As an indicator of the perceptual audio quality, we measure the objective difference grade of a colored noise audio signal emitted over a consumerlike hardware. The measurements show that the non-contiguous OQAM-OFDM scheme not only introduces lower interference to the FM link, but also has the advantage of requiring less number of notched carriers in comparison to CP-OFDM. By application of non-contiguous OQAM-OFDM with an appropriate number of notched carriers instead of the classical CP-OFDM scheme, wireless microphone systems can still sustain a significant low SIR when non-professional hardware is applied.
\end{abstract}

\section{INTRODUCTION}

In the past years, regulatory agencies have expedited changes in the regulations to satisfy the increasing demand for spectrum, as current and new customer related services require more bandwidth and reliable access. The most remarkable change was the digitalization of the terrestrial television broadcast (Digital Terrestrial Television - DTT). This opened up a lot of spectrum within the lower UHF bands in the range of 470 to $790 \mathrm{MHz}$ for new services. Due to its good RF propagation, these bands have gained lots of attraction for mobile service providers as well as for last-mile solutions [1]. Another opportunity to use unoccupied TV channels (TV white spaces - TVWS) is to dedicate them to the so-called TVWS devices in the context of spectrum sharing concepts, e.g. dynamic spectrum access (DSA) which is defined within the framework of cognitive radio [1]. Currently, the unoccupied TV channels are used by narrowband program making special event (PMSE) devices, which are operating in a quasilicensed manner. The drawback of this issue is the difficulty in affording coexistence.

For the improvement of coexistence, several approaches are currently investigated. The first one is to switch the TV band used by PMSE devices in case of detecting an interfering system [2]. This scheme is based on interference detection and avoidance before the PMSE system is disturbed. The second approach is to set a beacon in channels containing PMSE devices, which permits the access of any other TVWS devices, as currently considered in the IEEE 802.22 standard [3]. Another promising approach is the concept of spectrum pooling [4]. Therein broadband orthogonal frequency division multiplexing (OFDM) based systems, operating as secondary users, notch the carriers at frequencies currently occupied by primary or licensed user systems. This so-called non-contiguous OFDM (NC-OFDM) system concept can be beneficial in terms of increasing the efficiency in DSA scenarios [5] and thus is the subject of this paper.

The coexistence of two or more systems operating in the same frequency band has been object of several studies. Therein, the out-of-band (OOB) emission of different OFDM schemes and appropriate techniques for their reduction by appropriate pulse designs have been intensively investigated, e.g. in [6], [7]. Further studies on the performance of coexisting systems concerning the outage probability of the links are conducted on a system level for example in [8], [9].

Therefore, in this contribution we focus on the perceptual audio quality of PMSE links influenced by NC-OFDM systems as this is of crucial importance for all PMSE users as well as their vendors. The PMSE user is selected to be a common frequency modulation (FM) based wireless microphone (WM) system, since most of the currently deployed PMSE links are either WMs or in-ear-monitoring solutions using FM. Our objective is mainly to conceptualize the system designs which are necessary to achieve the maximum robustness in terms of perceptual audio quality, transmitted over the WM link, and its validation by measurements. In this study, for secondary user systems intended to coexist with the primary user-like operating WM, we use the classical cyclic-prefix OFDM (CP-OFDM), since most currently applied broadband systems use this modulation scheme, and the offset-QAM OFDM (OQAM-OFDM) scheme proposed for TVWS. Other modulation schemes with good power suppression, e.g. windowed OFDM or OFDM with cancellation carriers, are out of scope as we focus on modulation schemes with comparable spectral efficiency. In [10] and [11], the issue of DSA in the above mentioned OFDM schemes is described comprehensively. Beside a description of a system for coexistence 


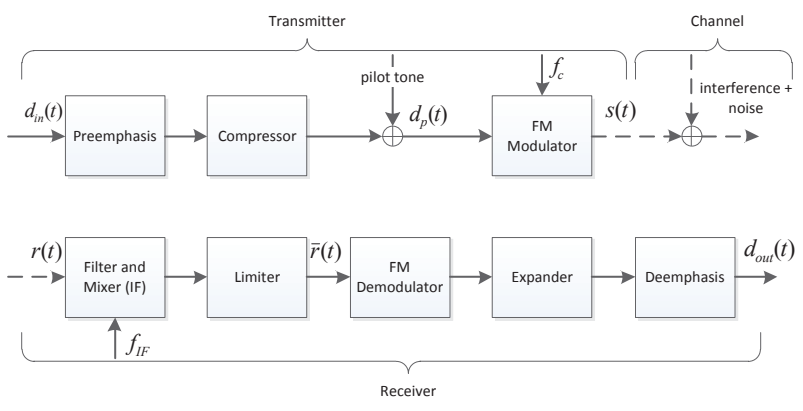

Fig. 1. Schematic illustration of a WM transmission link

of WMs with white space devices, [12] presents measurements for the influence of noise like interference imposed to commercially available WMs. To the best knowledge of the authors, a practical evaluation of the impact of NC-OFDM systems, including OQAM-OFDM, on a WM link has not been reported so far. Therefore we evaluate the performance of a WM system in a heterogeneous environment to cover this gap. We present experimental results, which quantify the achievable performance gain by application of the OQAMOFDM scheme [7] in comparison to CP-OFDM in terms of robustness and achievable throughput.

The remaining of this paper is structured as follows. Section II includes the description of the WM and OFDM systems as well as the deployed analysis algorithm, followed by the specification of the measurement setup in section III. The measurement results are summarized in section IV and the conclusion is presented in section $\mathrm{V}$.

\section{SYSTEM DESCRIPTION}

This section holds the descriptions of the investigated wireless microphone system and the multi-carrier (MC) broadband interferer systems as well as a description of the applied algorithm, namely perceptual evaluation of audio quality (PEAQ).

\section{A. Wireless Microphone System}

The microphone system consists of a unidirectional frequency modulated (FM) link as depicted in Fig. 1. With the frequency deviation $F$, carrier frequency $f_{c}$ and the amplitude $a_{0}$ the analytic FM signal $s(t)$ is described by

$$
s(t)=a_{0} e^{j\left(2 \pi f_{c} t+2 \pi F\left[\int_{0}^{t} d_{p}\left(t^{\prime}\right) d t^{\prime}\right]+\phi_{0}\right)} .
$$

Here, $\phi_{0}$ is a random initial phase of the carrier and $d_{p}(t)$ is a combination of the audio source signal $d_{\text {in }}(t)$ and a pilot tone at the frequency $f_{p}$ having initial phase $\phi_{p}$ and the frequency deviation of $F_{p}$

$$
d_{p}(t)=w\left(d_{\text {in }}(t)\right)+\frac{F_{p}}{F} \cos \left(2 \pi f_{p} t+\phi_{p}\right) .
$$

The function $w(\cdot)$ equates to the compression and preemphasis of the audio source. These operations are usually used in FM systems for increasing the signal-to-noise ratio (SNR) for quiet audio sections and audio frequencies above approximately $1 \mathrm{kHz}$.
TABLE I

\begin{tabular}{|c|c|}
\hline \multicolumn{2}{|l|}{ WM system } \\
\hline audio bandwidth $B_{d}$ & $22 \mathrm{kHz}$ \\
\hline frequency deviation audio $F$ & $48 \mathrm{kHz}$ \\
\hline frequency of pilot tone $f_{p}$ & $32.768 \mathrm{~Hz}$ \\
\hline frequency deviation pilot $F_{p}$ & $2 \mathrm{kHz}$ \\
\hline \multicolumn{2}{|c|}{ NC-OFDM systems } \\
\hline modulation order & 4-QAM \\
\hline number of subchannels (used) & $64(52)$ \\
\hline bandwidth & $10 \mathrm{MHz}$ \\
\hline subchannel spacing $\Delta f$ & $156.25 \mathrm{kHz}$ \\
\hline $\begin{array}{l}\text { cyclic prefix length (CP-OFDM) } \\
\text { (fractions of symbol duration) }\end{array}$ & 0.25 \\
\hline overlapping factor $K$ (OQAM-OFDM) & 4 \\
\hline prototype filter (OQAM-OFDM) & $\begin{array}{l}\text { as described in [7] with } \\
A[1]=0.97195983\end{array}$ \\
\hline
\end{tabular}

PARAMETERS OF THE WM AND NC-OFDM SYSTEMS

The audio source signal $d_{\text {in }}(t)$ is emulated with colored noise, which is generated by filtering white Gaussian noise with a filter circuit specified by ETSI [13] having the bandwidth $B_{d}$. By applying Carsons rule for bandwidth estimation of FM schemes the allocated bandwidth $B_{F M}$ of the presented microphone link is approximately $140 \mathrm{kHz}$, which has been confirmed by measurements (see Table II) and approximately corresponds to the width of one subcarrier spacing of the OFDM systems specified in Section II-C.

At receiver side the received signal $r(t)$ is filtered, down converted to the intermediate frequency (IF) $f_{I F}$ and afterwards limited to reduce noise, which can be approximated by

$$
\bar{r}(t)=\min \left(\mid g(t) *\left(r(t) e^{j\left(2 \pi\left(f_{I F}-f_{c}\right) t\right)} \mid\right), a_{\text {lim }}\right),
$$

where $g(t)$ represents the IF filter impulse response and $a_{\text {lim }}$ is the maximum output amplitude of the limiter. The audio signal $d_{\text {out }}(t)$ is obtained by demodulation with subsequent expansion and de-emphasis of the limited signal $\bar{r}(t)$. The main parameters of the WM link are listed in Table I.

\section{B. PEAQ}

For the quantitative rating of audio quality, physical, e.g. signal-to-noise ratio, or psychoacoustic, e.g. noise-to-mask ratio [14], measures can be taken into account. As the perceived auto quality by listeners to PMSE devices is affected by the human ear specific signal processing, this contribution focuses on a psychoacoustic analysis. Therefore we use the PEAQ algorithm, as it provides objective and reproducible results, contrary to the well known but subjective mean opinion score (MOS).

The PEAQ algorithm has been standardized by the ITU [15] and is part of the algorithm group PEXQ, which is used for the objective measurement of the experienced quality of transmitted information. Beside the evaluation of audio quality targeted by PEAQ, the PEXQ group contains algorithms for the objective quantification of speech, video as well as data transfer quality degradations. For the determination of the audio quality degradation, the PEAQ algorithm compares the received and distorted audio signal with the transmitted one 


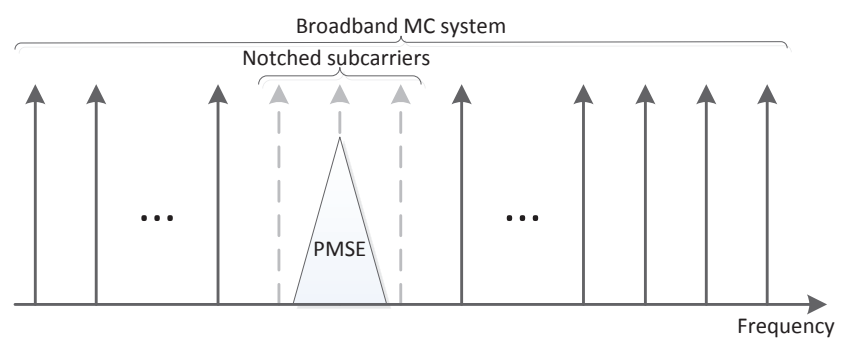

Fig. 2. Concept of WM in an OFDM spectrum pooling scenario [4]

by consideration of the human perception. The output of the PEAQ is the so-called objective difference grade (ODG) and ranges from 0 , representing imperceptible auditory degradation, up to -4 for very annoying audio quality. For comparison, these values can be mapped linearly to the MOS.

For the calculation of the correct ODG, the received $\left(d_{\text {out }}\right)$ and the reference audio signal $\left(d_{\text {in }}\right)$ have to be synchronized in time as well as normalized in power. In our investigations this is done by searching the maximum of the cross correlation between both digitized signals

$$
\hat{\tau}=\max _{\tau}\left(\sum_{L_{c}} d_{\text {in }}[l] d_{\text {out }}[l+\tau]\right),
$$

where $L_{c}$ represents the correlation window, followed by a normalization of the power of the received and synchronized audio signal $d_{\text {out }}[l+\hat{\tau}]$ to the power level of the reference signal as below:

$$
d_{\text {norm }}[l]=d_{\text {out }}[l+\hat{\tau}] \sqrt{\frac{\sum_{L}\left|d_{\text {in }}[l]\right|^{2}}{\sum_{L}\left|d_{\text {out }}[l+\hat{\tau}]\right|^{2}}},
$$

having $L$ audio samples. Afterwards, $d_{\text {norm }}[l]$ and $d_{\text {in }}[l]$ are fed into the PEAQ algorithm for the ODG calculation.

\section{Multi-carrier Systems}

In this work, two multi-carrier schemes based on the IEEE 802.11a (WiFi) standard [16] are utilized to evaluate the influence on FM based wireless microphone links. Even though conventional $\mathrm{WiFi}$ systems are operating in the industrial, scientific and medical (ISM) bands at $2.4 \mathrm{GHz}$ and $5 \mathrm{GHz}$, respectively, there are several efforts to adopt the system design for usage in the TV white space. Besides the experimental setup of WhiteFi [17], which is implemented by conversion of common WiFi signals to the UHF band, there are new approaching standards such as IEEE 802.11af and ECMA 392. These standards target at a WiFi-like connectivity for devices operating in TV the band and, from the physical layer point of view, only differ in terms of parameter settings. The systems considered in this work are based on the conventional design using CP-OFDM, as deployed in many commercial systems nowadays, and on the alternative approach of OQAMOFDM, respectively. A detailed description of both systems with the parameters specified in Table I, including simulated performance curves and a complexity analysis, is provided

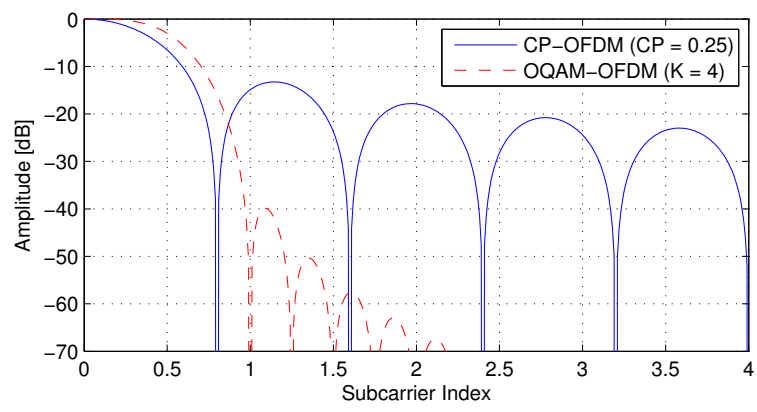

Fig. 3. Magnitude responses for the filters applied in the investigated MC schemes. The abscissa compression of the sinc-function of the CP-OFDM system is caused by the selected cyclic prefix length of 0.25 .

in [18]. The described designs are enhanced by an adaptive notching capability, which allows the MC signal to avoid the interfered spectrum parts in a circumfluent manner to account for the spectrum pooling concept as depicted in Fig. 2. This reduces the interference to the WM, which is mainly dependent on the magnitude response of the applied transmission filters, presented in Fig. 3, and the distance to the first allocated carrier. The discrete-time transmit signal $o[n]$ of a NC-OFDM system can be expressed by

$$
o[n]=\sum_{i=-\infty}^{\infty} \sum_{k \in K_{a}} d_{i, k} g_{k}\left[n-i T^{\prime}\right],
$$

where $d_{i, k}$ describes the complex QAM symbol and the real or imaginary part composing a QAM symbol on carrier index $k$ for the CP-OFDM and OQAM-OFDM scheme, respectively. The index $i$ is the symbol index. $T^{\prime}$ accounts for the different symbol spacing and is specified as $T^{\prime}=T$ for CP-OFDM and $T^{\prime}=T / 2$ for OQAM-OFDM modulation with $T$ equal the complex symbol duration before OQAM mapping. Furthermore, $K_{a}$ specifies the set of not notched data-bearing subchannels out of the complete set of subchannels $K$. The filter function $g_{k}[n]$ is defined as $g_{k}[n]=g[n] e^{-j 2 \pi \frac{k n}{K}}$ with $g[n]$ either being a rectangular window for the CP-OFDM case or with a filter function derived by the frequency sampling technique [7] and the parameters specified in Table I for OQAM-OFDM.

\section{Measurement Setup}

The measurement setup presented in Fig. 4 is used to determine the impact of different NC-OFDM interferer configurations on the perceptual quality of the audio signal. By using a wired setup we are capable of establishing defined and reproducible interference scenarios to the WM system. A reference audio signal is periodically and incessantly transmitted by a Sennheiser SK 2000 body pack transmitter, which has been altered to have a SMA antenna output port connector instead of its antenna. The transmitted FM signal was attenuated by $70 \mathrm{~dB}$ to operate close to the FM receiver sensitivity. This emulates a PMSE system which is most vulnerable to any occurring interference. Another advantage of a highly 


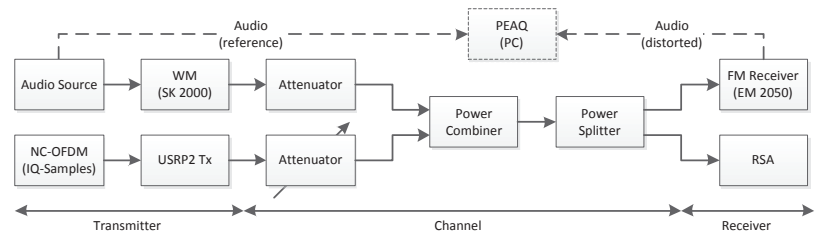

Fig. 4. Block diagram of the measurement setup applied for the ODG evaluation showing the FM link with a fixed power level as well as the different interfering links with variable power. A real-time spectrum analyzer (RSA) for monitoring and signal power measurements for SIR calculations is added.

TABLE II

MEASURED X DB BANDWIDTH OF THE WM SIGNAL WHERE X REPRESENTS THE ATTENUATION OF THE SIGNAL AT THE GIVEN BANDWIDTH.

\begin{tabular}{l|c|c|c|c|c|c|c} 
amplitude $[\mathrm{dB}]$ & -10 & -20 & -30 & -40 & -50 & -60 & -65 \\
\hline bandwidth $[\mathrm{kHz}]$ & 16.3 & 28.1 & 60 & 82.4 & 94.3 & 107 & 131
\end{tabular}

attenuated WM is the possibility to measure the influence of NC-OFDM systems with very good power suppression at the notched carriers. After attenuation the FM signal is fed into a power combiner to add the interfering NC-OFDM signals. Table II contains the measured spectral shaping of the generated FM signal. The NC-OFDM signals are generated by a Matlab implementation and transmitted by an USRP2 equipped with a WBX RF front end, which is capable of transmitting in the range from $50 \mathrm{MHz}$ to $2.2 \mathrm{GHz}$ and behaving similar to customer equipment. The multi-carrier signal strength is reduced by two programmable attenuators, each ranging from $0 \mathrm{~dB}$ to $31 \mathrm{~dB}$, and also fed into the power combiner so that we receive an interfered FM signal with a certain interference power level. Thereby, the amplification of the USRP2 is set to $20 \mathrm{~dB}$ to get a suitable power level with a limited non-linear operation of the amplifier. Fig. 5 presents sample spectrum plots for a WM as well as OQAM-OFDM and CP-OFDM systems with three notched carriers.

The combined signal is then distributed by a power splitter. One output is connected to a real-time spectrum analyzer (RSA), which is used to measure the signal-to-interference ratio (SIR) according to

$$
\mathrm{SIR}=\frac{\int_{\left(T_{m}\right)}|s(t)|^{2} d t}{\int_{\left(T_{m}\right)}|o(t)|^{2} d t},
$$

by deactivating either the WM transmitter or the OFDM system and measuring the power of each signal. Thereby $T_{m}$ is the measurement duration and $s(t)$ and $o(t)$ are defined according to (1) and (6), respectively. The second output is fed into a Sennheiser EM 2050 FM receiver for demodulation. The retrieved audio signal is sampled at a PC and afterwards analyzed by the PEAQ algorithm (basic mode) as described before. We use the PEAQ implementation provided and analyzed in [19].

Due to the filter bandwidth $B_{F}$ of $200 \mathrm{kHz}$ in the FM receiver, the above mentioned SIR measurement between both systems is of less significance, since the filter bandwidth is

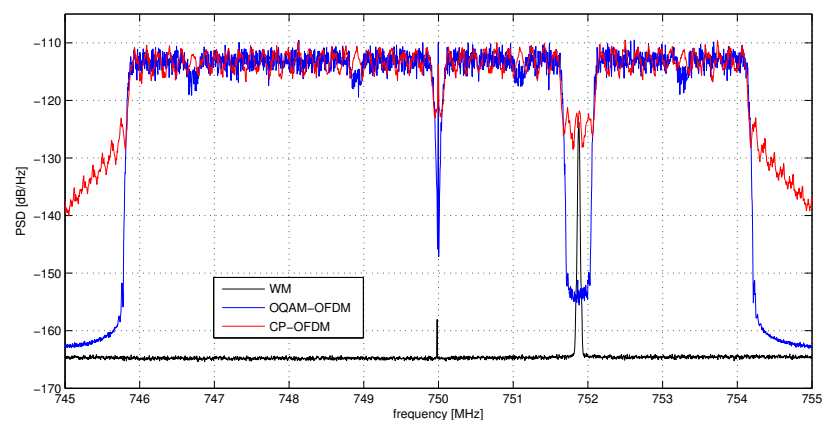

Fig. 5. Example spectrum plot (measured) for a WM (at $751.875 \mathrm{MHz}$ ) enclosed by an OQAM-OFDM system with three notched carriers. The estimated SIR between the WM and the NC-OFDM system is about $-37 \mathrm{~dB}$ $\left(\mathrm{SIR}_{\mathrm{PSD}} \approx-21 \mathrm{~dB}\right)$

TABLE III

PARAMETERS AND CONFIGURATION OF THE EXPERIMENTAL SETUP AS APPLIED FOR THE MEASUREMENTS. SUBCHANNEL \# 0 CORRESPONDS TO DC CARRIER.

\begin{tabular}{l|l}
\multicolumn{2}{c}{ Audio system } \\
\hline analog audio source & colored noise [13] \\
\hline bandwidth $B_{d}$ & $22 \mathrm{kHz}$ \\
\hline sampling frequency & $48 \mathrm{kHz}$ \\
\hline sampling resolution & $16 \mathrm{bit}$ \\
\hline \# of PEAQ frames & 1382 \\
\hline
\end{tabular}

Wireless microphone system

\begin{tabular}{l|l}
\hline transmitter / Receiver device & Sennheiser SK 2000 / EM 2050 \\
\hline center frequency $f_{c}$ & $751.875 \mathrm{MHz}$ \\
\hline microphone input level & $-24 \mathrm{dBV}$ \\
\hline microphone Sensitivity & $-30 \mathrm{~dB}$ \\
\hline microphone Tx power & $9.3 \mathrm{dBm}$ \\
\hline RF Attenuation & $70 \mathrm{~dB}$ \\
\hline Rx Filter bandwidth $B_{F}$ & $200 \mathrm{kHz}$ \\
\hline
\end{tabular}

\begin{tabular}{l|l}
\multicolumn{2}{c}{ OFDM systems } \\
\hline transmitter device & Ettus USRP2 \& WBX front-end \\
\hline center frequency $f_{\mathrm{DC}}$ & $750 \mathrm{MHz}$ \\
\hline sampling frequency & $10 \mathrm{MHz}$ \\
\hline$f_{c}$ of notch & $751.875 \mathrm{MHz}$ \\
\hline $3 \mathrm{~dB}$ bandwidth $B_{3 \mathrm{~dB}}$ & $8.2 \mathrm{MHz}$ \\
\hline USRP gain / tx power & $20 \mathrm{~dB} / 0 \mathrm{dBm}$ \\
\hline
\end{tabular}

Hardware for channel emulation

\begin{tabular}{l|l}
\hline WM attenuator & Agilent 8495B \\
\hline USRP2 attenuators & Mini Circuits ZX76-31-SP-S+ \\
\hline power combiner & Mini Circuits ZN2PD2-63-S+ \\
\hline power splitter & Mini Circuits ZN4PD1-63W-S+ \\
\hline
\end{tabular}

significantly smaller than the bandwidth of the OFDM systems, so that the measurement results will be mainly dependent on the bandwidth of the OFDM systems. For this reason we introduce the bandwidth independent $\mathrm{SIR}_{\mathrm{PSD}}$, which is defined as follows

$$
\mathrm{SIR}_{\mathrm{PSD}}=\frac{B_{3 \mathrm{~dB}}}{B_{F}} \cdot \mathrm{SIR} .
$$

Here, $B_{3 \mathrm{~dB}}$ is the $3 \mathrm{~dB}$ bandwidth of the OFDM system. Using Eq. (8) we obtain a reference value which can be easily applied for systems with different parameters, only depending on the difference of the average power spectral density (PSD) for the systems under comparison. 
TABLE IV

MEASURED AND SIMULATED PSD SUPPRESSION VALUES WITHIN A 200 KHZ CHANNEL FOR BOTH MC SYSTEMS DEPENDING ON THE NUMBER OF NOTCHED OFDM CARRIERS AND WITH THE PARAMETERS DEFINED IN TABLE III. FOR THE SIMULATIONS $10^{6}$ OFDM SYMBOLS CARRYING RANDOMLY DISTRIBUTED 4-QAM SYMBOLS HAVE BEEN USED.

\begin{tabular}{c||c|c|c|c}
\multicolumn{1}{c||}{} & \multicolumn{4}{c}{ Simulated } \\
\hline \# of notched carriers & 1 & 3 & 5 & 7 \\
\hline \hline CP-OFDM [dB] & 6.5 & 12.7 & 14.4 & 15.7 \\
\hline OQAM-OFDM [dB] & 5.9 & $\begin{array}{r}58.3 \\
\text { Measured }\end{array}$ & 75.1 & 83.7 \\
\hline \# of notched carriers & 1 & 3 & 5 & 7 \\
\hline \hline CP-OFDM [dB] & 5.9 & 11.6 & 12.9 & 13.4 \\
\hline OQAM-OFDM [dB] & 6.1 & 42 & 42 & 42
\end{tabular}

\section{RESULTS AND DisCUSSION}

The results presented in this section are obtained by applying the parameters and configuration listed in Table III. This section is divided into two parts. To obtain the audio link quality measure, the power suppression in the subchannel occupied by the WM in dependence of the number of notched carriers is evaluated in the first section. The second part focuses on the influence of the power suppression performance on the perceptual audio quality.

\section{A. Power Suppression}

The measurement results of the power suppression are listed in Table IV. It can be seen that notching one carrier provides only a slight power suppression of about $6 \mathrm{~dB}$ for both systems. This can be explained by the fact that the main lobes of both OFDM systems are still very strong at the edges of the $200 \mathrm{kHz}$ channels (see Fig. 3). In addition, the interference caused by the CP-OFDM system is increased by a significant influence of its first side lobe.

For an increased number of notched carriers, the power suppression of the CP-OFDM system increases only very slowly, since the sinc-function decreases only proportional to $1 /(\Delta f)$. Additionally the transmission hardware decreases the notching performance of CP-OFDM slightly up to about $2 \mathrm{~dB}$ due to imperfections inherited by the system components.

As indicated by Fig. 3, the measurement results show that the OQAM-OFDM system performs significantly better than CP-OFDM. There is an increase in power suppression up to $30 \mathrm{~dB}$, which is less than that delivered the simulations. This gain reduction can be assigned to the imperfections and limitations of the used USRP2 and its RF front-end, e.g. amplifier non-linearities and noise figure.

Although limitations of the customer-like hardware significantly degrade the performance of power reduction in the OQAM-OFDM scheme, it still performs about $30 \mathrm{~dB}$ better than CP-OFDM if more than one subcarrier is notched. With this result we recommend non-contiguous OQAM-OFDM for application in spectrum pooling scenarios containing FM based PMSE devices, as the influence of an OQAM-OFDM system is considerably lower than for CP-OFDM based systems.

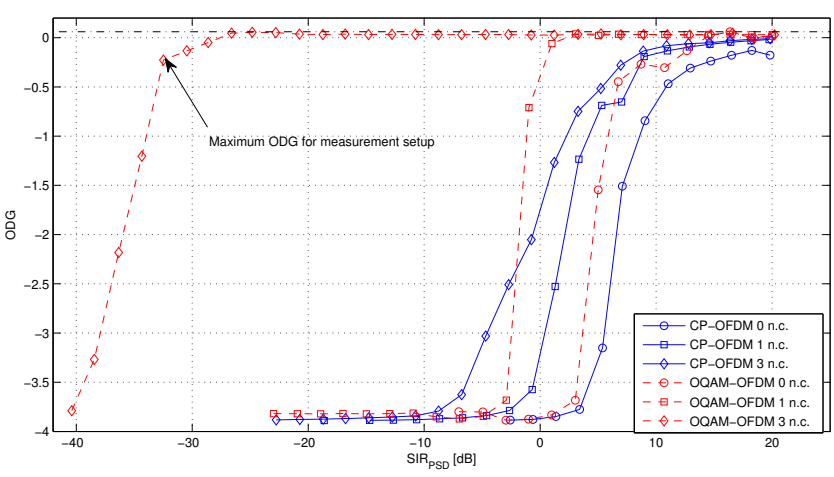

Fig. 6. ODG for a WM audio link interfered by different OFDM systems and amount of notched carriers (n.c.) relative to their PSD difference SIR $P$ SD. The black dash-dotted line is the maximum ODG achievable with the measurement setup (0.06).

\section{B. Perceptual Audio Quality}

For the PEAQ analysis of the measurement setup we concentrate on the notching of one and three carriers, respectively, since we want to keep the throughput degradation of the OFDM systems as low as possible. Furthermore, the power suppression of the OQAM-OFDM system is limited and the gain in power suppression shows only a marginal increase for more than three notched subcarriers as described before. In the following evaluation we focus on the ODG range of 0 to -2 as these values represent audio quality without any perceptible up to tolerable disturbances. The measurement results are presented in Fig. 6, whereby the ODG of the used reference audio stream $\left(d_{\text {norm }}[l] \equiv d[l]\right)$ is 0.19 and the ODG of the undisturbed and attenuated FM link is 0.06 . These values indicate, that the presented measurement setup is permissible for our investigations, as the complete audio transmission chain imposes only inaudible distortions.

By notching the subcarrier located at the position of the $\mathrm{WM}$, the robustness gain in $\mathrm{SIR}_{\mathrm{PSD}}$ is about 3.5 to $5 \mathrm{~dB}$ for CP-OFDM and 6 to $8 \mathrm{~dB}$ for OQAM-OFDM, although both systems experience an equivalent power suppression. The difference between both systems of about $2 \mathrm{~dB}$ can be explained by the spectral shaping of the OFDM systems (see Fig. 3) as well as the used FM modulation of the wireless microphone (see Table II). As the colored noise, which has been used to emulate the audio signal, has the largest power density in the lower frequency parts up to $1 \mathrm{kHz}$, most of the information is transferred close to the carrier of the FM signal. For the CP-OFDM system the first side lobes of the adjacent carriers have a large power contribution at the center of the FM signal, which decreases the ODG compared to the OQAMOFDM system. Here, the main power contribution is close to the edge of the FM channel, as the side lobes experience a much larger attenuation compared to the CP-OFDM system. This resistance of the FM signal against interference at the its channel edges is additionally increased by the pre-emphasis applied to the audio signal, which increases the robustness in higher frequencies within the audio signal against distortions. 
TABLE V

ROBUSTNESS GAIN (SIR PSD) OBTAINED BY CARRIER NOTCHING IN COMPARISON TO A CORRESPONDING CONTIGUOUS OFDM SYSTEM.

\begin{tabular}{l||c|c||c|c}
\multicolumn{1}{c||}{} & \multicolumn{2}{c||}{ CP-OFDM } & \multicolumn{2}{c}{ OQAM-OFDM } \\
\hline ODG & 1 n.c. & 3 n.c. & 1 n.c. & 3 n.c. \\
\hline \hline 0 (imperceptible) & $3.5 \mathrm{~dB}$ & $5.5 \mathrm{~dB}$ & $7 \mathrm{~dB}$ & $39.5 \mathrm{~dB}$ \\
\hline-1 (perceptible, not annoying) & $4 \mathrm{~dB}$ & $6.5 \mathrm{~dB}$ & $6.5 \mathrm{~dB}$ & $40 \mathrm{~dB}$ \\
\hline-2 (slightly annoying) & $4.5 \mathrm{~dB}$ & $8.5 \mathrm{~dB}$ & $6 \mathrm{~dB}$ & $41 \mathrm{~dB}$
\end{tabular}

TABLE VI

ROBUSTNESS GAIN (SIR PSD $_{\text {B }}$ B APPLICATION OF OQAM-OFDM INSTEAD OF CP-OFDM.

\begin{tabular}{l||c|c|c} 
ODG & 0 n.c. & 1 n.c. & 3 n.c. \\
\hline \hline 0 & $4.5 \mathrm{~dB}$ & $8 \mathrm{~dB}$ & $38.5 \mathrm{~dB}$ \\
\hline-1 & $2 \mathrm{~dB}$ & $4.5 \mathrm{~dB}$ & $35.5 \mathrm{~dB}$ \\
\hline-2 & $2 \mathrm{~dB}$ & $3.5 \mathrm{~dB}$ & $34.5 \mathrm{~dB}$
\end{tabular}

In case of three notched carriers only the magnitude response side lobes of the OFDM systems affect the bandwidth allocated by the WM. Table V contains the values of the SIR $_{P S D}$ gain compared to the continuous OFDM schemes. For OQAM-OFDM the achieved gain values are approximately the same as the power suppression values discussed in section IV-A, slightly depending on the amount of acceptable audio distortions. The gain for CP-OFDM is about 1 to $6 \mathrm{~dB}$ less than indicated by the power suppression values of Table IV, since the center of the second side lobe of the magnitude response is at the same position as the center frequency of the WM.

Comparing the performance of contiguous OQAM-OFDM with CP-OFDM, as listed in Table VI, it can be conducted that PMSE devices are about 2 to $4 \mathrm{~dB}$ more robust against the OQAM-OFDM system than for CP-OFDM systems. By application of subcarrier notching, the gain in robustness can be increased by 4 to $8 \mathrm{~dB}$ for one notched subcarrier and 35 to $39 \mathrm{~dB}$ for three notched subcarriers, respectively. In general the robustness is increased by about $44 \mathrm{~dB}$ in comparison to the currently applied CP-OFDM schemes and the proposed non-contiguous OQAM-OFDM with three notched carriers.

\section{CONCLUSion}

In this work, we compared the degradation in perceptible audio quality of a WM link, interfered by different NC-OFDM schemes. We verified by measurements that the spectrum pooling concept can be used to improve the coexistence between wireless microphones, as a part of the PMSE device group, and OFDM based systems. By comparing with contiguous CP-OFDM, the robustness can be improved by approximately $44 \mathrm{~dB}$ at comparable perceptual audio quality level by applying the non-contiguous OQAM-OFDM scheme with three carriers notched. In conclusion, the coexistence of narrowband wireless microphones and overlayed broadband multi-carrier systems can be significantly improved and the overall spectral efficiency can be increased.

\section{ACKNOWLEDGMENT}

This work was supported by Sennheiser electronic GmbH \& Co. KG.

\section{REFERENCES}

[1] Q. Zhao and A. Swami, "A Survey of Dynamic Spectrum Access: Signal Processing and Networking Perspectives," in 2007 IEEE International Conference on Acoustics, Speech and Signal Processing - ICASSP '07, no. May. IEEE, 2007, pp. IV-1349-IV-1352.

[2] Uwe Beutnagel-Buchner, N. Hilbich, and A. Wilzeck, "C-PMSE Improved Spectrum Utilization and Coexistence by Cognitive PMSE Systems: A strategic application-oriented research \& development project in Germany," 6th International ICST Conference on Cognitive Radio Oriented Wireless Networks and Communications, pp. 296-300, 2011.

[3] I. S. Association, "IEEE Standard for Information Technology Telecommunications and information exchange between systems - Local and metropolitan area networks - Specific requirements Part 22.1: Standard to Enhance Harmful Interference Protection for Low-Power Licensed Dev," pp. 1-145, 2010.

[4] T. A. Weiss and F. K. Jondral, "Spectrum Pooling: An Innovative Strategy for the Enhancement of Spectrum Efficiency," Communications Magazine, IEEE, no. March, pp. 8-14, 2004.

[5] L. Yang, W. Hou, L. Cao, B. Y. Zhao, and H. Zheng, "Supporting Demanding Wireless Applications with Frequency-agile Radios," in 7th USENIX conference on Networked systems design and implementation, 2010, pp. 1-15.

[6] T. Weiss, J. Hillenbrand, A. Krohn, and F. Jondral, "Mutual interference in OFDM-based spectrum pooling systems," in 2004 IEEE 59th Vehicular Technology Conference. VTC 2004-Spring (IEEE Cat. No.04CH37514), vol. 4. IEEE, 2004, pp. 1873-1877.

[7] A. Viholainen, T. Ihalainen, T. H. Stitz, M. Renfors, and M. Bellanger, "Prototype Filter Design for Filter Bank Based Multicarrier Transmission," in 17th European Signal Processing Conference (EUSIPCO), 2009, pp. 1359-1363.

[8] Z. Zhao, M. Schellmann, H. Boulaaba, and E. Schulz, "Interference Study for Cognitive LTE-Femtocell in TV White Spaces," in Technical Symposium at ITU Telecom World 2011 ITU WT11, 2011, pp. 153-158.

[9] K. W. Sung, L. Shi, and J. Zander, "Coexistence of LTE femtocells with GSM cellular network," in 21st Annual IEEE International Symposium on Personal, Indoor and Mobile Radio Communications. IEEE, Sep. 2010, pp. 1556-1560.

[10] B. Farhang-Boroujeny, "OFDM Versus Filter Bank Multicarrier," IEEE Signal Processing Magazine, vol. 28, no. 3, pp. 92-112, May 2011.

[11] D. Noguet, M. Gautier, and V. Berg, "Advances in opportunistic radio technologies for TVWS," EURASIP Journal on Wireless Communications and Networking, vol. 2011, no. 1, p. 170, 2011.

[12] G. Nychis, R. Chandra, T. Moscibroda, I. Tashev, and P. Steenkiste, "Reclaiming the white spaces: spectrum efficient coexistence with primary users," in Proceedings of the Seventh COnference on emerging Networking EXperiments and Technologies, ser. CoNEXT'11. ACM, 2011, pp. 1-12.

[13] ETSI, "EN 300 422-1 V1.3.2 - Electromagnetic compatibility and Radio spectrum Matters (ERM); Wireless microphones in the $25 \mathrm{MHz}$ to 3 $\mathrm{GHz}$ frequency range; Part 1: Technical characteristics and methods of measurement," pp. 1-41, 2008.

[14] J. Johnston, "Estimation of Perceptual Entropy Using Noise Masking Criteria," in ICASSP-88., International Conference on Acoustics, Speech, and Signal Processing. IEEE, 1988, pp. 2524-2527.

[15] ITU-R, "RECOMMENDATION ITU-R BS.1387-1 - Method for objective measurements of perceived audio quality," pp. 1-100, 2001.

[16] "Supplement to IEEE Standard for Information Technology - Telecommunications and Information Exchange Between Systems - Local and Metropolitan Area Networks - Specific Requirements. Part 11: Wireless LAN Medium Access Control (MAC) and Physical Layer (PHY)," IEEE Std 802.11a-1999, p. i, 1999.

[17] P. Bahl, R. Chandra, T. Moscibroda, R. Murty, and M. Welsh, "White Space Networking with Wi-Fi like Connectivity," ACM SIGCOMM Computer Communication Review, vol. 39, no. 4, pp. 1-12, Aug. 2009.

[18] M. Fuhrwerk, C. Thein, and L. Häring, "Performance Comparison of CP-OFDM and OQAM-OFDM Based WiFi Systems," in Proceedings of the 16th International OFDM-Workshop (InOWo'11), Hamburg, 2011, pp. 11-15.

[19] P. Kabal, "An Examination and Interpretation of ITU-R BS. 1387: Perceptual Evaluation of Audio Quality," Tech. Rep., 2003. 